obese had an OR of $1.40(95 \%$-CI $1.35-1.45$; p for trend $<0.001)$ for any injury or pain in the lower limbs compared to children and adolescents with normal body weight after adjustments for sex, age, race/ethnicity, and socioeconomic factors. Similarly, extremely obese children and adolescents had increased relative odds for: fractures (OR 1.26, 95\%-CI 1.20-1.33); sprains/strains (OR 1.47, 95\%-CI 1.36-1.59), dislocations (OR 1.41, 95\%-Cl 1.36-1.47), and pain (OR 1.45, 95\%-CI 1.24-1.70). For all injuries except dislocations, the odds ratios increased with increasing degree of overweight/ obesity ( $\mathrm{p}$ for trend $<0.001$ ), with underweight children/adolescents having lower relative odds of all injuries and pain compared to the normal weight group. Conclusions: Overweight, obese, and extremely obese children/ adolescents are more likely to experience lower extremity injuries and/or pain than are their underweight and normal weight peers. While the precise mechanism underlying these associations remains unclear, lower extremity injuries can reduce the physical activity in weight groups that need to increase activity levels to reduce or manage their weight.

Keywords: Obesity, Childhood, Injuries

doi:10.3121/cmr.2011.1020.c-c2-02

Plenary III-01:

HMORN Research on Pediatric Hypertension and Obesity: Predictors, Care, and Costs: Design of a New Pediatric Cohort

Kenneth Adams, $\mathrm{PhD}^{1}$; Emily Parker, $\mathrm{PhD}, \mathrm{MPH}^{1}$; Nicole Schneider, $\mathrm{BA}^{1}$; Heather Tavel, BS ${ }^{2}$; Malini Chandra, $\mathrm{MS}^{2}$; Mary Becker, BA'; Maureen Peterson, $\mathrm{BA}^{1}$; Karen Margolis, $\mathrm{MD}, \mathrm{MPH}^{1}$; Nancy Sherwood, $\mathrm{PhD}^{1}$; Elyse Kharbanda, MD, $\mathrm{MPH}^{1}$; Alan Sinaiko, $\mathrm{MD}^{3}$; David Magid, $\mathrm{MD}, \mathrm{MPH}^{2}$; Matthew Daley, MD, MPH${ }^{2}$; Joan Lo, $\mathrm{MD}^{2}$; Patrick O'Connor, MD, $\mathrm{MPH}^{1}$

${ }^{1}$ Health Partners Research Foundation; ${ }^{2}$ Kaiser Permanente; ${ }^{3}$ University of Minnesota Medical

Background: The increasing numbers of children or adolescents having elevated blood pressure (BP) is an important public health problem, but little information is available regarding patterns of care or resource use implications in this population. We established a data cohort of children and adolescents to study these relationships. Methods: We have implemented a longitudinal cohort study based on routinely collected electronic medical record data from 3 large health plans. The study will ultimately extend from calendar year 2007 through 2012. We have currently extracted subject data through 2009. We will continue to accrue new subjects through 2012 and to add data for existing subjects in future years. The source population consists of children and adolescents (3-17 yr) enrolled in the 3 health plans. Beginning in Jan 2007, age-eligible subjects enter the cohort at the time of the first clinic visit in which an eligible BP is measured (i.e., collected during an outpatient visit and associated with a corresponding height measure). Subjects are being followed forward in time with longitudinal data collection. Demographic, clinical, and administrative data include age, gender, race/ethnicity, BP, height, weight, diagnoses, medications, and cost of care. We have conducted preliminary analyses to demonstrate the accrual of subjects into the cohort over years 2007 through 2009, and describing the gender, age, and racial composition of the study population. Results: As of the end of 2009, the cohort included 330,880 children and adolescents. At entry, 58.1\% were $3-11$ years of age, whereas $41.9 \%$ were $12-17$ years. Subjects were $50.2 \%$ female. Race was $41.0 \%$ white (combining Hispanics and non-Hispanics), $8.1 \%$ African-American, $12.8 \%$ Asian/Pacific Islander, $16.3 \%$ other, and $21.8 \%$ missing. Conclusions: We have created a large pediatric cohort of subjects using data collected from health plan electronic medical and administrative records at several HMORN sites. These de-identified data include longitudinal BP, BMI, and utilization data suitable to test hypotheses related to correlation of BP and BMI over time, the influence of BMI on BP, patterns of care provided to those with elevated $\mathrm{BP}$ and $\mathrm{BMI}$, and the influence of elevated $\mathrm{BP}$ and BMI on resource use.

Keywords: Predictors, Electronic medical records, Pediatric hypertension doi:10.3121/cmr.2011.1020.plenary-iii-01
C-C2-04:

\section{Diabetic Patients and Risk - It's Not About the Numbers}

Barry Saver, MD, $\mathrm{MPH}^{1}$; Kathleen Mazor, EdD ${ }^{1}$; Lee Hargraves, $\mathrm{PhD}^{2}$

${ }^{1}$ UMass Medical School/Meyers Primary Care Institute; ${ }^{2}$ UMass Medical School

Background: The major, common adverse health outcomes for patients with diabetes come from macrovascular, not microvascular, disease, including death, heart attack and stroke. Personalized risk information might help patients make better decisions about prioritizing health behavior change. In this study, we explored diabetic subjects' beliefs about diabetes-related risks and sources of information along with reactions to presentation of personalized risk information. Methods: We recruited 56 English- and Spanish-speaking adults with diabetes and at least one other major cardiovascular risk factor from a community health center and an academic family medicine practice. In in-depth interviews, we explored sources of risk information, riskreduction activities, and behavioral intentions. Subjects also ranked 6 diabetes-associated adverse outcomes based on perceived risk, reviewed personalized risk predictions from the UKPDS Outcomes Model, and re-ranked these perceived risk of outcomes. We explored their reasons for changing/not changing risk rankings. Qualitative analysis was used to develop themes and concepts underlying subjects' risk perceptions and reactions to risk information. Results: Virtually all subjects believed at least some diabetes-related adverse outcomes were modifiable or preventable. A substantial minority expressed a fatalistic view that at least some were not preventable, while others felt that risk factor control meant an outcome was "not going to happen." A very common theme was that a "warning shot" would occur before many outcomes, providing time and impetus to change bad habits. Provider urging was the other commonly-cited motivator and about $20 \%$ of subjects cited each as motivators for past change. While providers were cited as a key source of information, vicarious experience with relatives and acquaintances was cited just as often and $3 / 4$ of subjects felt they knew themselves and their risks better than providers or risk models. Subjects reported the personalized risk information was interesting, but $<1 / 3$ changed their rankings of mortality (nearly always initially ranked least likely) to match the model predictions (nearly always the highest probability). Conclusions: Our subjects often based their risk perceptions on anecdote and gut feelings, not medical information. Personalized risk information does not appear promising for motivating behavior change or even altering risk perceptions.

Keywords: Diabetes, Risk perception

doi:10.3121/cmr.2011.1020.c-c2-04

\section{C-B5-05:}

Early Childhood Obesity Prevention in Primary Care: Opt-In Versus Opt-Out Recruitment Strategies

Margaret Rukstalis, $\mathrm{MD}^{1}$; Chelsie Hauer, $\mathrm{BA}^{1}$

${ }^{1}$ Geisinger Health System

Background: Although early childhood obesity prevention programs are needed in primary care to address the growing pediatric obesity epidemic, recruitment remains particularly challenging.AimsTo describe opt-in versus opt-out recruitment strategies to invite parents of overweight/obese 4-8 year olds to participate in a family lifestyle modification obesity prevention study in primary care. Methods: Following IRB approval, traditional recruitment strategies were employed including flyers, doctor referrals, and public ads to invite parents of 4-8 year olds with a Body Mass Index (BMI) $>85$ th percentile for age and sex to call (Opt-IN) about participating in a 5-month primary care obesity prevention intervention. Electronic Health Records were searched and physician letters were sent to parent/guardian of eligible children who resided at zip codes within 1-hour commute of treatment site to invite them to call about the study. Opt In letters were mailed over 13-months for Cohorts 1-3 recruitment. For Cohort 4, identical physician letters were mailed except parents were asked to call if they were NOT interested in information about the study (Opt-OUT). After 10 days, study staff called to 